\title{
Bovine arch anatomy influences recoarctation rates in the era of the extended end-to-end anastomosis
}

\author{
Joseph W. Turek, MD, PhD, ${ }^{a}$ Brian D. Conway, MD, MS, ${ }^{a}$ Nicholas B. Cavanaugh, BS, ${ }^{a}$ \\ Alex M. Meyer, BS, ${ }^{\mathrm{a}}$ Osamah Aldoss, MD, ${ }^{\mathrm{b}}$ Benjamin E. Reinking, MD, ${ }^{\mathrm{b}}$ Ahmed El-Hattab, MD, ${ }^{\mathrm{a}}$ and \\ Nicholas P. Rossi, MD
}

\section{ABSTRACT}

Objectives: Arch branching has never been shown to influence recoarctation after extended end-to-end anastomosis via thoracotomy, yet in each study bovine arch identification is grossly underreported. This study aims to (1) assess chart review reliability in bovine arch identification; (2) determine recoarctation risk with a bovine arch; and (3) explore an anatomic explanation for recurrent arch obstruction based on arch anatomy.

Patients: A total of 49 consecutive patients underwent thoracotomy with extended end-to-end aortic coarctation repair at a single institution (2007-2012).

Methods: Echocardiograms from these patients were reviewed for arch anatomy and compared with the echocardiographic reports. Recurrent arch obstruction was defined as an echocardiographic gradient across the repair of $20 \mathrm{~mm} \mathrm{Hg}$ or greater. For cases with angiographic images $(n=17)$, a scaled clamping distance between the left subclavian artery and the maximal proximal clamp location on orthogonal projections was then calculated across arch anatomies.

Results: Chart review identified 6.1\% (3/49) of patients with a bovine arch compared with $28.6 \%$ (14/49) on targeted image review. A total of $28.6 \%$ (4/ 14) of patients with a bovine arch had a follow-up gradient of $20 \mathrm{~mm} \mathrm{Hg}$ or greater. Only 5.7\% (2/35) of patients with normal arch branching had a followup gradient of $20 \mathrm{~mm} \mathrm{Hg}$ or greater. The mean clamping index was significantly diminished in patients with bovine arch anatomy.

Conclusions: Arch anatomy often goes undocumented on preoperative imaging, yet children undergoing extended end-to-end repair with bovine arch anatomy are at a significantly increased risk of recoarctation. This may be due to a reduced clampable distance to facilitate repair. These results should be considered in the preoperative assessment, parental counseling, and surgical approach for children with discrete aortic coarctation. (J Thorac Cardiovasc Surg 2018;155:1178-83)

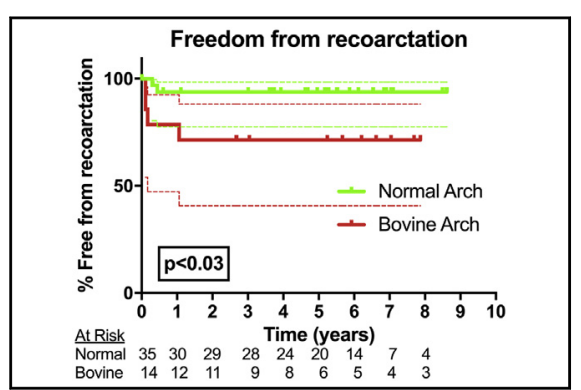

Bovine arch branching has higher recoarctation rates after extended end-to-end repair.

\section{Central Message}

Bovine arch anatomy predisposes to aortic recoarctation after extended end-to-end anastomosis via a left thoracotomy. This may be due to a shortened clampable distance on the arch.

\section{Perspective}

Arch anatomy often goes undocumented on preoperative imaging for coarctation. Yet, the presence of a bovine arch carries an increased risk of recoarctation for infants and neonates undergoing extended end-to-end repair through a left thoracotomy. These results should alter the paradigm for preoperative assessment, parental counseling, and surgical approach for children with discrete aortic coarctation.

See Editorial Commentary page 1184

See Editorial page 1176.

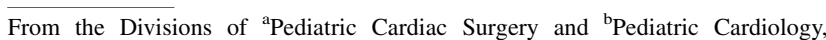
University of Iowa Children's Hospital, Iowa City, Iowa.

Read at the 97th Annual Meeting of The American Association for Thoracic Surgery, Boston, Massachusetts, April 29-May 3, 2017.

Received for publication May 23, 2017; revisions received Sept 11, 2017; accepted for publication Oct 14, 2017; available ahead of print Nov 29, 2017.

Address for reprints: Joseph W. Turek, MD, PhD, Division of Cardiovascular and Thoracic Surgery, Duke University School of Medicine, Duke Children's Hospital, 2301 Erwin Rd, DUMC 3474, Durham, NC 27710 (E-mail: joseph.turek@duke. edu).

$0022-5223 / \$ 36.00$

Copyright (c) 2017 by The American Association for Thoracic Surgery

https://doi.org/10.1016/j.jtcvs.2017.10.055
The repair of discrete aortic coarctation has been described by numerous operative techniques, including end-to-end anastomosis, patch aortoplasty, and subclavian flap aortoplasty. ${ }^{1-4}$ Each of these repair strategies is traditionally performed through a left thoracotomy. However, these

Scanning this QR code will take you to the article title page. 
techniques have largely fallen out of favor because of concerns of higher reintervention rates, not to mention stunted ipsilateral arm growth in patients with subclavian flap aortoplasty. ${ }^{5}$ The extended end-to-end anastomosis has emerged as the procedure of choice when approaching a coarctation via a left thoracotomy. This technique has consistently demonstrated lower recoarctation rates than the aforementioned predecessors and advantageously addresses distal arch hypoplasia. ${ }^{6-9}$

Several investigations have looked at predictors of recoarctation with the extended end-to-end approach. The only identified risk factors for recoarctation from this body of work include proximal arch hypoplasia, age less than 2 weeks, and weight less than $2 \mathrm{~kg}{ }^{10,11}$ As such, many groups have advocated for a median sternotomy approach using a variety of end-to-side repair techniques to address these particular risk factors. ${ }^{12-15}$ The common brachiocephalic trunk, commonly referred to as a "bovine arch," remains notably absent from this group of risk factors, because only a few of the extended end-to-end studies assessed arch anatomy in relation to reintervention after coarctation repair. Furthermore, those investigations asking this question concluded that bovine arch anatomy had no statistical influence on recoarctation. However, on more detailed review, the cohort prevalence of bovine arch anatomy in these studies was only $3 \%$ to $5 \% .^{10,16,17}$ Meanwhile, the true prevalence of a bovine arch is more likely in the $15 \%$ to $37 \%$ range, as reported in imaging and autopsy analysis. ${ }^{18-20}$ Thus, we propose the consequence of bovine arch anatomy in coarctation repair has never been adequately assessed, and echocardiographic reports lack attention to arch anatomy assessment.

This study aimed to (1) assess chart review reliability in bovine arch identification; (2) determine the recoarctation risk with a bovine arch after extended end-to-end repair via a left thoracotomy; and (3) explore an anatomic explanation for recurrent arch obstruction based on arch anatomy.

\section{PATIENTS AND METHODS \\ Patient Population and Data Collection}

The University of Iowa Institutional Review Board approved this study before the collection of data. The need for individual patient consent was waived. We retrospectively reviewed the records of 49 consecutive children undergoing surgical repair for aortic coarctation via an extended end-to-end anastomosis at a single institution over a 6-year period (2007-2012). A secured database was constructed for the cohort detailing patient and operative characteristics, such as age, weight, gender, associated genetic conditions, presence of a hypoplastic distal arch, and aortic clamp time, during the operation. A hypoplastic proximal arch was defined as an aortic diameter just distal to the left common carotid artery of less than the child's kilogram of body weight +1 (in millimeters).

The database was also used to detail arch branching anatomy from chart reviews and targeted image analysis. Finally, echocardiographic gradients across the repair at the corresponding time of follow-up were recorded in the database.

\section{Identification of Arch Anatomy}

A single pediatric cardiologist, blinded to the patient's previously documented arch anatomy, specifically reviewed the preoperative echocardiographic images for all 49 patients for arch anatomy (normal or bovine arch). Consistent with prior publications, bovine branching anatomy was defined as a common aortic arch origin of the left common carotid artery and innominate artery or the left common carotid artery originates as a branch of the innominate artery. ${ }^{18-21}$ These results were compared with a chart review of the preoperative echocardiographic reports looking for the same.

\section{Operative Technique}

All operations were performed through a posterolateral left thoracotomy, with entry into the chest cavity via the third or fourth intercostal space. The aortic arch was fully mobilized from just distal to the innominate artery to well beyond the area of coarctation on the descending thoracic aorta. After heparinization, the proximal clamp was placed on the arch just beyond the innominate trunk, including control of the left common carotid and subclavian arteries. Distal vascular control was attained by clamping the descending aorta distant from the coarctation site. The patent ductus arteriosus (or ligamentum arteriosum) was ligated on the side of the pulmonary artery. The aorta was then transected adjacent to the coarctation site, and all ductal tissue was removed. The underside of the transverse arch was then incised maximally toward the proximal clamp. The distal aorta was then incised laterally to extend and match the opening to the proximal aorta. The extended end-to-end anastomosis was then performed with a running monofilament suture.

\section{Evaluation of Recoarctation}

Recurrent arch obstruction was determined by transthoracic echocardiographic reports with a gradient across the repair site $20 \mathrm{~mm} \mathrm{Hg}$ or greater. These gradients were compared across follow-up periods and across arch anatomies.

\section{Assessment of Clampable Distance}

For cases with angiographic images (17/49; 6 bovine arches; 11 normal arches), a scaled clamping distance (indexed to the diameter of the sinotubular junction) between the left subclavian artery and the maximal proximal clamp location on orthogonal projections was then calculated for normal anatomy (Figure 1, $A$ ) versus bovine arch anatomy (Figure 1, B).

\section{Statistical Analysis}

Variables were compared between patients with a normal arch and patients with a bovine arch. Statistical analysis was performed using GraphPad Prism 7 (GraphPad Software Inc, La Jolla, Calif). Data were assessed for normality using a Shapiro-Wilk test. Normally distributed data are presented as mean \pm standard deviation, and non-normally distributed data are presented as median with range. Categoric variables are presented as frequencies and percentages. Crude associations for categoric variables were evaluated through the use of a Fisher exact test. For continuous variables, median differences were evaluated with a Wilcoxon Mann-Whitney test, including a test for equality of variances or a Student $t$ test as appropriate. Freedom from recoarctation was analyzed by Kaplan-Meier curve analysis using Mantel-Cox log-rank test.

\section{RESULTS}

\section{Echocardiographic Arch Anatomy Assessment}

Review of echocardiographic reports identified only 3 of 49 patients $(6.1 \%)$ with documentation of bovine arch (or equivalent nomenclature) anatomy. Meanwhile, targeted echocardiographic image analysis by a single pediatric 

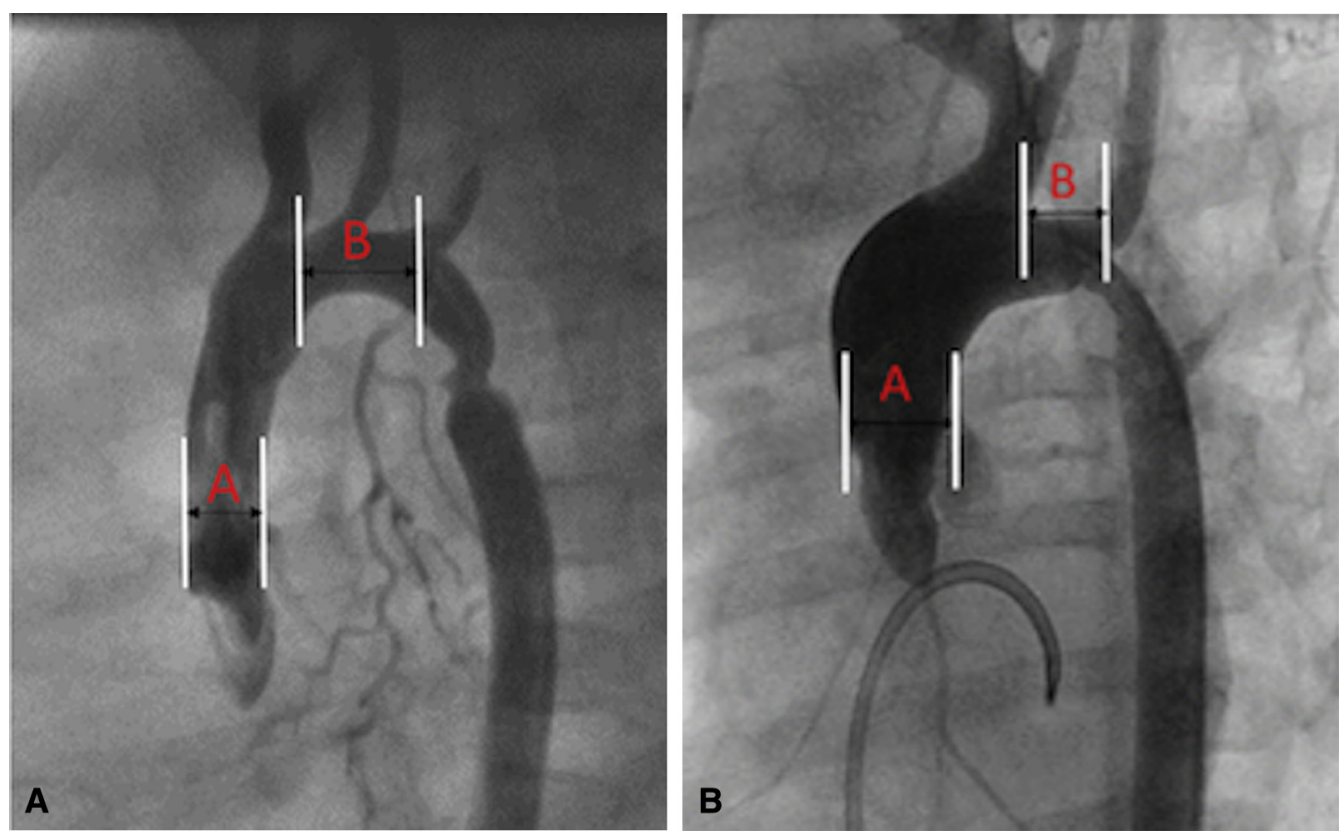

FIGURE 1. Measurement of clamping index from angiographic images for a normal arch (A) and bovine arch (B). The clamping index provides a normalized assessment of the maximal transverse aortic arch available to facilitate extended end-to-end coarctation repair via a left thoracotomy. "B" denotes the clampable distance from immediately after the brachiocephalic trunk to the proximal side of the left subclavian artery. "A" measures the aortic diameter at the sinotubular junction. The clamping index is defined as the ratio of $\mathrm{B} / \mathrm{A}$.

cardiologist classified 14 of 49 (28.6\%) arches as possessing bovine anatomy (Figure 2). The latter frequency compares more favorably with imaging and autopsy assessments of bovine arch prevalence. ${ }^{18-20}$

\section{Patient and Operative Characteristics}

Table 1 displays patient demographics, diagnoses, and aortic clamp times as stratified by arch anatomy determined by direct image analysis. None of the variables measured showed any significant differences between the normal arch and bovine arch cohorts.

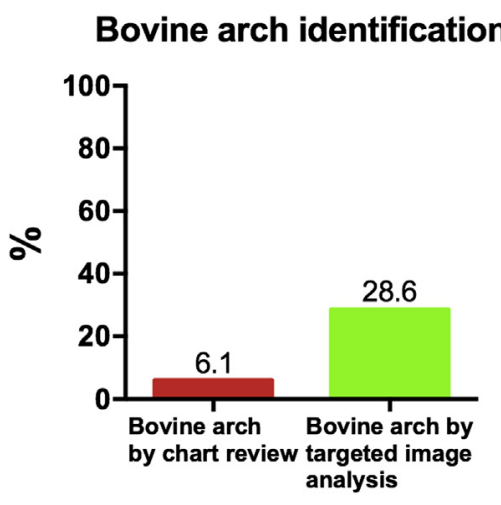

FIGURE 2. Bovine arch is underreported on preoperative echocardiographic reports. Chart review of our cohort revealed only $6.1 \%$ prevalence of a bovine arch. Targeted echocardiographic image analysis for arch anatomy exposed a $28.6 \%$ prevalence of a bovine arch in the same cohort.

\section{Recurrent Arch Obstruction}

Over the period of the study, 6 of the 49 patients had echocardiographic evidence of recoarctation, with a gradient of $20 \mathrm{~mm} \mathrm{Hg}$ or greater across the repair site. Recurrent arch obstruction for normal arch anatomy was $5.7 \%(2 / 35)$, whereas patients with a bovine arch experienced recoarctation in $28.5 \%$ (4/14). All of these patients with recurrent arch obstruction underwent balloon angioplasty, with none requiring stenting or repeat operation to date. Two of the bovine arch recoarctations subsequently required a second balloon angioplasty procedure. Median duration of follow-up echocardiography for the entire cohort was 3.73 years (range, 0.01-8.62 years). Meanwhile, median follow-up echocardiography duration was 3.96 years (range, 0.01-8.62 years) for patients with normal arch anatomy and 3.17 years (range, 0.01-7.87 years) for patients with bovine arch anatomy $(P=.39)$. Figure 3 demonstrates Kaplan-Meier curve calculation for freedom from recoarctation. With the use of the Mantel-Cox log-rank test, there was significantly higher recoarctation rates in the cohort with bovine arch anatomy $(P<.03)$. Of note, all recurrent arch obstructions in the entire cohort occurred in infants (4/11 bovine arch cases; 2/24 normal arch cases).

\section{Maximal Clamping Assessment}

Angiographic images were available for 17 (6 bovine arches; 11 normal arches) of the 49 patients in the series preoperatively or within 7 months postoperatively. 
TABLE 1. Patient and operative characteristics

\begin{tabular}{llcc}
\hline \multicolumn{1}{c}{ Variable* } & $\begin{array}{c}\text { Normal arch } \\
(\mathbf{n}=\mathbf{3 5})\end{array}$ & $\begin{array}{c}\text { Bovine arch } \\
(\mathbf{n}=\mathbf{1 4})\end{array}$ & $\begin{array}{c}\boldsymbol{P} \\
\text { value }\end{array}$ \\
\hline Demographics & & & \\
$\quad$ Age, $\mathrm{d}$ & $96(4-5176)$ & $40.5(6-2645)$ & .47 \\
$\quad$ Weight, kg & $10.3 \pm 10.1$ & $7.6 \pm 7.5$ & .37 \\
$\quad$ Male sex & $24(68.6)$ & $9(64.3)$ & .76 \\
Diagnosis & & & \\
$\quad$ Genetic syndrome & $2(5.7)$ & 0 & .36 \\
$\quad$ Hypoplastic arch & $5(14.3)$ & $2(14.2)$ & .99 \\
Operation & & & \\
$\quad$ Clamp time, min & $23(12-39)$ & $21(7-40)$ & .38 \\
\hline
\end{tabular}

*Continuous variables are displayed as median (range) or mean \pm standard deviation as appropriate; categoric variables are presented as frequency (percentage).

Clamping indices, as described in Figure 1, were significantly lower in the presence of a bovine arch $(0.80 \pm 0.39)$ than with a normal arch $(1.31 \pm 0.55)$ $(P<.050)$.

\section{DISCUSSION}

As often occurs in medicine, this study was borne out of a clinical suspicion based on an anecdotal experience. Specific to this investigation, a coarctation repair via a left thoracotomy was particularly difficult because of a limited transverse arch between the crossclamps. This, in a patient with a known bovine arch, prompted the question: Does a bovine arch predispose to recoarctation? The congenital literature is abundant with studies looking at risk factors for recoarctation in the era of the extended end-to-end anastomosis. However, there exists a paucity of work evaluating arch anatomy as a variable. Even in these rare studies accounting for arch anatomy, a bovine arch has never been

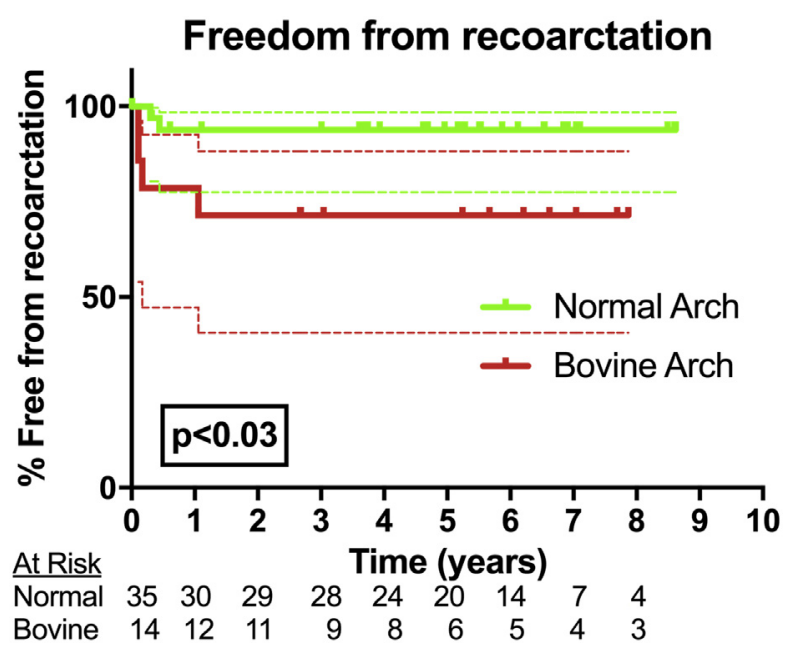

FIGURE 3. Kaplan-Meier curves depicting freedom from recoarctation (dark lines with dashed lines delineating 95\% confidence intervals) for patients with normal or bovine arch. A bovine arch negatively influences recoarctation rates in the era of extended end-to-end anastomosis $(P<.03)$. found to influence reintervention or recoarctation. ${ }^{10,16,17}$ Deeper analysis into the data reveals only a $3 \%$ to $5 \%$ prevalence of bovine arch anatomy in these articles. Meanwhile, a host of imaging and autopsy studies suggest the true prevalence of a bovine arch to be in the $15 \%$ to $37 \%$ range. ${ }^{18-20}$ Thus, we propose that the consequence of bovine arch anatomy in coarctation repair has never been adequately assessed.

\section{Identification of Arch Anatomy}

The term "bovine arch," although used interchangeably with common brachiocephalic trunk, bicarotid trunk, and 2vessel arch, represents a misnomer when describing human anatomy. ${ }^{21}$ In fact, the typical cow's arch consists of a single branch containing both carotid and subclavian vessels. Nonetheless, when used to describe human aortic arch branching, a bovine arch is assumed to represent either of 2 bicarotid trunk variants. The first is characterized by the left common carotid artery sharing arch origin with the innominate artery. The second variant consists of the left common carotid artery arising from the innominate artery distinct from the arch.

The aforementioned discrepancy in bovine arch prevalence between retrospective chart reviews and directed imaging and autopsy studies was recapitulated in our work. Specifically, our institutional echocardiographic reports (and likely the echocardiographic evaluation itself) lacked attention to arch anatomy assessment, noting a bovine arch in only $6.1 \%$. Targeted echocardiographic image analysis of the same cohort revealed $28.6 \%$ prevalence of a common brachiocephalic trunk. As such, chart reviews to elucidate arch branching remain insufficient. This is possibly a function of the perceived inconsequential nature of arch branching variants. The demonstration in this article that bovine arch anatomy does influence recoarctation rates should systematically change the way preoperative echocardiographic assessments are performed for coarctation of the aorta. In particular, arch anatomy should be documented as a mandatory part of the examination.

\section{The Bovine Arch and Implications for Extended End- to-End Repair}

A bovine arch is often described as the left common carotid artery arising more proximally on the arch, so as to originate immediately adjacent to or as a branch of the innominate artery. Although it is likely the left common carotid artery is displaced more proximally in bovine arch anatomy, it is also plausible that the innominate artery is also displaced more distally on the arch. This latter anatomic subtlety would act to shorten the length of the transverse arch and can thereby impede the ability to perform a true extended end-to-end anastomosis. In fact, a memorable coarctation repair in a patient with bovine arch anatomy performed through a left thoracotomy was 
particularly challenging because of the limited available transverse arch once the clamps were applied. This experience became the impetus for initiating this study. Our angiographic analysis comparing "clamping indices" between bovine arches $(0.80 \pm 0.39)$ and normal branching arches $(1.31 \pm 0.55)(P<.050)$ supports this notion. The significant differences noted in clamping indices were all a function of an attenuated clampable arch in the bovine anatomy, because sinotubular junction measurements were indistinguishable between the normal and bovine arch variants. Of note, both preoperative and postoperative angiograms could be used for these calculations because the outer curvature measured in the clamping distance is not affected by the coarctation repair occurring largely on the underside of the arch.

The effects of diminished arch length to facilitate coarctation repair would be expected to be more pronounced in smaller patients. In fact, all recoarctations in the current study, both in the normal arch branching and the bovine arch anatomy cases, occurred in infants (aged $<1$ year). Furthermore, 5 of the 6 recoarctations were in neonates (aged $<30$ days). This follows, because the foreshortened clampable distance essentially converts an extended endto-end anastomosis to an end-to-end repair, which is more at risk for recurrent arch obstruction in smaller children.

From an institutional standpoint, these results have changed our management of discrete aortic coarctation. If the echocardiogram clearly demonstrates a bovine arch in a neonate (or a small infant), we proceed with repair via a median sternotomy using cardiopulmonary bypass. If there exists uncertainty regarding arch anatomy, we use computed tomography angiography, again with identified bovine arch cases receiving a repair by means of a sternotomy and normal arch cases undergoing a left thoracotomy. It has been our experience that bovine arches are consistently shorter with regard to clamping index, and thus we have not used actual clamping indices to guide our approach clinically. In addition, we are just now developing norms for clamping indices based on computed tomography images for normal and bovine arches, which may allow better stratification as to what constitutes an adequate clamping distance. Nonetheless, the future use of computed tomography angiography guidance to estimate the viability of a successful repair via a left thoracotomy remains a possibility.

\section{Study Limitations}

The retrospective design of this study serves as a limitation. This is particularly true in chart reviews assessing for recoarctation. The definitive assessment of arch anatomy in our study was based on direct echocardiographic image analysis, rather than chart review of echocardiographic reports. In comparing the 2 , we exposed the underreporting of bovine arch prevalence in both our hands and in the literature. This portion of our study underscores the drawbacks of retrospective chart review and suggests why a bovine arch has never before been shown to influence recoarctation rates. Finally, although this study demonstrated significance in recoarctation rates between arch anatomies, the modest cohort size (49 patients) could benefit from validation with a larger dataset.

\section{CONCLUSIONS}

Bovine arch anatomy often goes undocumented on preoperative imaging assessment, yet children undergoing extended end-to-end repair with bovine arch anatomy are at a significantly increased risk of recurrent arch obstruction. This may be due to a reduced clampable distance to facilitate repair. These results should be considered in the preoperative assessment, parental counseling, and surgical approach for children with discrete aortic coarctation. We recommend targeted echocardiographic assessment of arch branching anatomy for all cases of aortic coarctation. Furthermore, we espouse a median sternotomy approach for neonates and small infants with bovine arch anatomy, even with only a discrete aortic coarctation.

\section{Conflict of Interest Statement}

Authors have nothing to disclose with regard to commercial support.

\section{References}

1. Crafoord C, Nylin G. Congenital coarctation of the aorta and its surgical treatment. J Thorac Surg. 1945;14:347-61.

2. Gross RE, Hufnagel CA. Coarctation of the aorta. Experimental studies regarding its surgical correction. N Engl J Med. 1945;233:287-93.

3. Vosschulte K. Surgical correction of coarctation of the aorta by an "isthmusplastic" operation. Thorax. 1961;16:338-45.

4. Waldhausen JA, Nahrwold DL. Repair of coarctation of the aorta with a subclavian flap. J Thorac Cardiovasc Surg. 1966;51:532-3.

5. Shenberger JS, Prophet SA, Waldhausen JA, Davidson WR Jr, Sinoway LI. Left subclavian flap aortoplasty for coarctation of the aorta: effects on forearm vascular function and growth. J Am Coll Cardiol. 1989;14:953-9.

6. Amato JJ, Rheinlander HE, Cleveland CJ. A method of enlarging the distal transverse arch in infants with hypoplasia and coarctation of the aorta. Ann Thorac Surg. 1977;23:261-3.

7. Lansman S, Shapiro AJ, Schiller MS, Ritter S, Cooper R, Galla JD, et al. Extended aortic arch anastomosis for repair of coarctation in infancy. Circulation. 1986;74(3 Pt 2):I37-41.

8. Brown JW, Fiore AC, King H. Isthmus flap aortoplasty: an alternative to subclavian flap aortoplasty for long-segment coarctation of the aorta in infants. Ann Thorac Surg. 1985;40:274-9.

9. Backer CL, Mavroudis C, Zias EA, Amin Z, Weigel TJ. Repair of coarctation with resection and extended end-to-end anastomosis. Ann Thorac Surg. 1998; 66:1365-71.

10. McElhinney DB, Yang S-G, Hogarty AN, Gleason MM, Zachary CH, Rome JJ, et al. Recurrent arch obstruction after repair of isolated coarctation of the aorta in neonates and young infants: is low weight a risk factor? J Thorac Cardiovasc Surg. 2001;122:883-90.

11. Bacha EA, Almodovar M, Wessel DL, Zurakowski D, Mayer JE, Jonas RA, et al. Surgery for coarctation of the aorta in infants weighing less than $2 \mathrm{~kg}$. Ann Thorac Surg. 2001;71:1260-4.

12. Karl TR, Sano S, Brawn W, Mee RB. Repair of hypoplastic or interrupted aortic arch via sternotomy. J Thorac Cardiovasc Surg. 1992;104:688-95.

13. Dodge-Khatami A, Backer CL, Mavroudis C. Risk factors for recoarctation and results of reoperation: a 40-year review. J Card Surg. 2000;15:369-77.

14. Mery CM, Guzman-Pruneda FA, Carberry $\mathrm{KE}$, Watrin $\mathrm{CH}$, McChesney GR, Chan JG, et al. Aortic arch advancement for aortic 
coarctation and hypoplastic aortic arch in neonates and infants. Ann Thorac Surg. 2014;98:625-33.

15. Nellis JR, Chung TK, Agarwal N, Torres JE, Holgren SE, Raghavan ML, et al. Modeling outcomes: modified aortic arch advancement for neonatal hypoplastic arch. Innovations. 2017;12:109-15.

16. Van Son JA, Falk V, Schneider P, Smedts F, Mohr FW. Repair of coarctation of the aorta in neonates and young infants. J Card Surg. 1997;12:139-46.

17. Burch PT, Cowley CG, Holubkov R, Null D, Lambert LM, Kouretas PC, et al. Coarctation repair in neonates and young infants: is small size or low weight still a risk factor? J Thorac Cardiovasc Surg. 2009; 138:547-52.

18. de Garis CF, Black IH, Riemenschneider EA. Patterns of the aortic arch in American white and negro stocks, with comparative notes on certain other mammals. J Anat. 1933;67:599-619.
19. Berko NS, Jain VR, Godelman A, Stein EG, Ghosh S, Haramati LB. Variants and anomalies of thoracic vasculature on computed tomographic angiography in adults. J Comput Assist Tomogr. 2009;33:523-8.

20. Reinshagen L, Vodiskar J, Muhler E, Hovels-Gurich HH, Vazquez-Jimenez JF Bicarotid trunk: how much is "not uncommon?" Ann Thorac Surg. 2014;97: 945-9.

21. Layton KF, Kallmes DF, Cloft HF, Lindell EP, Cox VS. Bovine aortic arch variant in humans. clarification of a common misnomer. Am J Neuroradiol. 2006;27: 1541-2.

Key Words: bovine arch, coarctation of the aorta, congenital heart disease, outcomes, pediatric 